Alan R. Camina

Nagoya Math. J.

Vol. 53 (1974), 47-57

\title{
FINITE GROUPS OF CONJUGATE RANK 2
}

\author{
ALAN R. CAMINA
}

\section{Introduction}

In $1953 \mathrm{~N}$. Itô defined the conjugate rank of a finite group as the number of distinct sizes, not equal to 1 , of the conjugacy classes of the group [7].

Let $G$ be a finite group and let $\left\{n_{1}, \cdots, n_{r}\right\}$ be a set of integers

$$
n_{i}>1 \forall i, \quad n_{i}=n_{j}
$$

if and only if $i=j$; and such that every conjugacy class in $G$ has $n_{i}$ elements for some $i$ and for every $i, n_{i}$ is the size of some conjugacy class of $G$, then $r$ is the conjugate rank of $G$.

N. Itô showed that any group of conjugate rank 2 is soluble [8]. It is the purpose of this paper to strengthen this result.

A group $G$ is called a group of type $F$, or of isolated type, if for every pair

$$
x, y \in G ; x, y \notin Z(G), C_{G}(x) \cap C_{G}(y)=Z(G) \text { or } C_{G}(x)=C_{G}(y) .
$$

R. Schmidt and J. Rebmann have completely classified such groups $[9,10]$. The main theorem is the following:

THEOREM 2. Let $G$ be a finite group of conjugate rank 2 which is not isolated. Then $G$ is a direct product of an Abelian group and a group whose order involves no more than 2 primes.

Combining this with the work of Rebmann and the theorem of Burnside on groups whose orders are divisible by just 2 primes, Itô's theorem follows from Theorem 2. The proof of this depends on the main theorem of [3] and on an extension of this which is proved in section 3. Unhappily in the earlier paper the situation described by $\left(^{*}\right)$ is not sufficiently general for the application intended. The following is

Received June 28, 1973. 
the required situation which is also needed as a basis for the proof of Theorem 2.

(**) Let $G$ be a finite group containing a proper subgroup $A$ satisfying the following conditions:

(i) $A=C_{G}(x)$ for some $x \in G$,

(ii) $\exists$ an integer $r$ such that if $y \in G$ and $C_{G}(y)<A$ then $\left[A: C_{G}(y)\right]=r$.

(iii) There exists no element $z \in G$ satisfying

$$
A<C_{G}(z)<G \text {. }
$$

Let $\pi(A)=\{p \mid p$ is a prime such that $\exists$ a $p$-power element whose centralizer is $A$ \}.

Then $A$ satisfies the condition that every non-central element of $A$ which has order prime to some element of $\pi(A)$ has index $r$ in $A$. The index of an element is defined to be the number of elements in the conjugacy class containing it. In [3] the main theorem classified groups satisfying the above condition when $|\pi(A)|>1$. In this paper a similar result is obtained for the situation when $|\pi(A)|=1$. The result is stated as a corollary, as it is obtained as a consequence of a slightly more general theorem.

COROLlaRY. Let $G$ be a finite group, $p$ a prime number and $n$ an integer. If every $p^{\prime}$-element of $G$ has 1 or $n$ conjugates and $|G| Z(G) \mid$ is divisible by at least two primes different from $p$, then $G$ is soluble with p-length $\leqslant 2, q$-length $=1, q$ a prime different from $p$ and $n$ is a power of $p$.

It is convenient at this point to define for any group $G$ a $G$-eccentric prime $p$ to be a prime such that $G / Z(G)$ has a non-trivial $p$-element. If $q$ divides $|G|$ and $q$ is not $G$-eccentric then the Sylow $q$-subgroup of $G$ is an Abelian direct factor of $G$. We can now state the theorem.

THEOREM 1. Let $G$ be a finite group such that the set of G-eccentric primes divides into two disjoint subsets, $\pi$ and $\sigma$, and let $n$ be an integer

Further let $G$ satisfy the following conditions:

(i) $G$ has a non-trivial nilpotent Hall o-subgroup

(ii) every non-central $\pi$-element of $G$ has $n$ conjugates, and

(iii) $|\pi|>1$.

Then $G$ is soluble, $n$ is a $\sigma$-number, and $G=O_{\pi}(G) \times Z_{0}$ where $Z_{0}$ is 
a central $\{\pi, \sigma\}$-subgroup of $G$. Further either:

(1) $G$ has a normal Abelian Hall $\pi$-subgroup $L_{0}$ such that $G / C_{G}\left(L_{0}\right)$ acts regularly on $L_{0} / L_{0} \cap Z(G)$, or

(2) $O_{\pi}(G) \leqslant Z(G)$ and $L / O_{\pi}(G)(Z(G) \cap L)$ is cyclic and $O_{\sigma \pi \sigma}(G) / L$ acts regularly on $L / O_{n}(G)(Z(G) \cap L)$, where $L=O_{\sigma \pi}(G)$.

The corollary follows by applying Theorem 1 with $\sigma=\{p\}$ and $\pi=$ $\{G$-eccentric primes excluding $p\}$. The term regularly will be used in the sense of D. Gorenstein [5] to mean that each element acts fixed point freely. This avoids the possible confusion of using fixed point freely in two different senses.

In the second section a number of trivial but useful lemmas are proved and a very useful proposition which may well be of independent interest.

Proposition 1. Let $G$ be a finite group with a subgroup $A_{0}$ such that $A_{0}$ is a characteristic subgroup of $A$, a subgroup of $G$, such that every element of $A_{0}$ has centralizer $A$ or $G$. Let $\pi$ be the set of primes dividing $\left|A_{0} / A_{0} \cap Z(G)\right|$ and assume $|\pi|>1$. Then either

(i) $N_{G}(A) / A$ is a $\pi^{\prime}$-group or,

(ii) $\left|N_{G}(A) / A\right|=p$ for some $p \in \pi$.

It is clear that this proposition could be used to give alternative proofs of the theorems concerning a group of type $F$ [9], [10].

Notation. Most of the notation is standard, see for example [5] or [6]. Let $G$ be a finite group. $p$ is said to be a $G$-eccentric prime if $p|| G / Z(G) \mid$. If $x \in G, \operatorname{Ind}_{G}(x)=\left[G: C_{G}(x)\right]=$ the order of the conjugacy class containing $x$. If $A<G$ and $x \in A$ then to say that $x$ is non-central will usually mean that $x \notin Z(G)$. For a definition of conjugate rank see [7].

\section{Preliminary Lemmas and a Useful Proposition}

We begin with a series of simple lemmas which contain results which are frequently used in the analysis of this type of problem.

LEMMA 1. Let $q$ be a prime dividing the order of $G$.

(i) If $x$ is in $G$ and $\operatorname{Ind}_{G}(x)=\operatorname{Ind}_{G}(y)$ for some non-central $q$ element $y$, then $C_{G}(x)$ contains non-central q-elements.

(ii) If $q$ divides $\operatorname{Ind}_{G}(w)$ for all non-central q-elements $w$ then 
$Z(Q) \leqslant Z(G)$ where $Q$ is a Sylow q-subgroup of $G$.

Proof. (i) Let $q^{a}$ be the highest power of $q$ which divides $\operatorname{Ind}_{G}(x)$. Let $Q_{0}$ be a Sylow $q$-subgroup of $C_{G}(x)$. If (i) is false $Q_{0} \leqslant Z(G)$. Let $Q$ be a Sylow $q$-subgroup of $G$ such that $Q \cap C_{G}(y)$ is a Sylow $q$-subgroup of $C_{G}(y)$. Now $\left[Q: Q_{0}\right]=q^{a}$ and $C_{Q}(y) \geqslant\left\langle y, Q_{0}\right\rangle$. Thus $\left[Q: C_{Q}(y)\right]<q^{a}$ which contradicts the assumption that $\operatorname{Ind}_{G}(x)=\operatorname{Ind}_{G}(y)$.

(ii) If $x \in Z(Q), \operatorname{Ind}_{G}(x)$ is prime to $q$. Thus $x \in Z(G)$.

LEMma 2. Let $A$ be a proper subgroup of $G$ which is the centralizer of an element in $G$, and let $\pi$ be a set of at least two G-eccentric primes each of which divides the order of $A$. If the centralizer of each $\pi$-element of $A$ has order $|G|$ or $|A|$ then $A$ possesses an Abelian Hall $\pi$-subgroup. Further if there is a $\pi$-element whose centralizer is $A$ then the Hall $\pi$-subgroup is central in $A$.

Proof. Let $p$ and $q$ be G-eccentric primes in $\pi$ and let $x$ and $y$ be non-central $p$ - and $q$-elements of $A$ respectively. Then $x y$ is a noncentral $\pi$-element lying in $A$. Thus $\left|C_{G}(x y)\right|=|A|$. But $C_{G}(x y)=C_{G}(x)$ $\cap C_{G}(y)$ and $\left|C_{G}(x)\right|=\left|C_{G}(y)\right|=|A|$. So we conclude that $C_{G}(x)=C_{G}(y)$ $=C_{G}(x y)$. The two results now follow.

LEMMA 3. Let $G$ be a group with a normal nilpotent subgroup $H$ which has a nilpotent complement $K$ of coprime order such that for all $x, y \in K \backslash C_{K}(H), C_{H}(x)=C_{H}(y)$. Then $K / C_{K}(H)$ is cyclic or a direct product of a cyclic group with a generalised quaternion group.

Proof. It is only necessary to show that there is some group on which $K$ acts regularly. Consider $\left(C_{H}(x)\right)^{H}$ for any $x \in K \backslash C_{K}(H)$. Since $H$ is nilpotent, $\left(C_{H}(x)\right)^{H}<H$ and clearly $K / C_{K}(H)$ acts regularly on $H /\left(C_{H}(x)\right)^{H}$.

Lemma 4. Let $P$ be an Abelian p-group, for some prime $p$ and let $K$ be a group of automorphisms of $P$ whose order is divisible by $p$. If for all pairs $x, y \in K \backslash\{1\}, C_{P}(x)=C_{P}(y)$ then $O_{p^{\prime}}(K)=1$.

Proof. Put $H=O_{p^{\prime}}(K)$ and assume $H \neq 1$. Since $K$ is not a $p$ group, $C_{P}(H) \neq 1$. Hence by a simple extension of the proof of Maschkes Theorem, $P=C_{P}(H) \times L$ where $L$ is a $K$-invariant subgroup of $P$. However $K$ would have to act regularly on $L$ and this is false since $K$ is not a $p^{\prime}$-group. 
The proof of Proposition 1 will be deduced from a sequence of Lemmas which will prove some stronger results than Proposition 1. It is convenient to assume a slightly weaker hypothesis to begin with.

(B) Let $A_{0} \leqslant A \leqslant G$ be a sequence of finite groups where $A_{0}$ is characteristic in $A$. Let $\pi$ be the set of $G$-eccentric primes dividing $\left|A_{0}\right|$. Finally assume the following three conditions:

(a) If $x \in A_{0}$ then $C_{G}(x)=A$ or $G$

(b) $|\pi|>1$.

(c) $N_{G}(A) \neq A$.

Lemma 5. Let $A_{0} \leqslant A \leqslant G$ satisfy (B). If $X \leqslant A_{0}$ and $X$ is not central, $C_{G}(X)=A$ and $N_{G}(X) \leqslant N_{G}(A)$.

Proof. Let $x \in X \backslash Z(G)$. Then $C_{G}(x)=C_{G}(X)=A . \quad C_{G}(X) \triangleleft N_{G}(X)$, and so $A \triangleleft N_{G}(X)$.

Lemma 6. Let $A_{0} \leqslant A \leqslant G$ satisfy (B). Let $W=N_{G}(A) / A$. Then

(i) if $U \leqslant W$ and $U$ is a $p^{\prime}$-group for some $p \in \pi$ then $U$ acts regularly on some section of $A_{0}$;

(ii) every Sylow subgroup of $W$ is cyclic or generalized quaternion;

(iii) any Sylow $q$-subgroup of $W$, for $q \in \pi$, has order $q$.

Proof. (i) Let $P_{0}$ be a Sylow $p$-subgroup of $A_{0}$. Since $A_{0}$ is characteristic in $A, P_{0}$ is characteristic in $A_{0}$ and so $U$ acts on $P_{0}$. Since $\left(|U|,\left|P_{0}\right|\right)=1$ and $C_{P_{0}}(u)=P_{0} \cap Z(G)$ for all $u \neq 1, u \in U, U$ acts regularly on $P_{0} \mid P_{0} \cap Z(G)$.

(ii) This follows immediately from (i) and $|\pi|$ being greater than one.

(iii) Let $V$ be a Sylow $q$-subgroup of $W$ and $Q_{0}$ be a Sylow $q$-subgroup of $P_{0} . \quad V$ acts faithfully on $Q_{0}$ and $C_{Q_{0}}(V)=Q_{0} \cap Z(G)$. Let $T$ be a subgroup of $Q_{0}$ such that $\left|T / Q_{0} \cap Z(G)\right|=q$ and $[T, V] \leqslant Q_{0} \cap Z$. Such a subgroup exists because $Q_{0}$ and $V$ are $q$-groups. From Lemma 5 it follows that $V$ acts faithfully on $T$ but $V / C_{V}(T)$ is elementary Abelian. From (ii) we know that if $V$ has exponent $q$ it is cyclic and so (iii) is proven.

Lemma 7. Let $A_{0} \leqslant A \leqslant G$ satisfy $(B)$. Then $N_{G}(A) / A$ is a $\pi$ or $\pi^{\prime}$-group. If $N_{G}(A) / A$ is a $\pi$-group then $\left|N_{G}(A) / A\right|=p$ for some prime $p \in \pi$. 
Proof. Let $W=N_{G}(A) / A$. If $W$ is soluble there exist subgroups of order $r s$ for any pairs of primes $r, s$ dividing $|W|$, from Lemma 6 (ii). If $W$ is not soluble then the Sylow 2-subgroup of $W$ is quaternion, again from Lemma 6 (ii), and so by [1] the involution in $W$ is central. Thus we have subgroups of order $2 r$ for any prime $r$ dividing $|W|$.

Let $U$ be a subgroup of $r s$ for two primes dividing $|W|$ where at least one of the pair is in $\pi$. If one is not in $\pi, U$ will be Abelian by Lemma 6 (i) and $[6 ; \mathrm{V} .8 .12]$. Thus we can assume that $U$ has a normal $r$-complement for $r \in \pi$. Let $R_{0}$ be the Sylow $r$-subgroup of $A_{0}$, which is clearly normalized by $U$. Hence by Lemma $4, U$ has no normal $r^{\prime}$-subgroup which is false. Hence $U$ does not exist and so either $W$ is a $\pi^{\prime}$ group or is a $p$-group for some prime $p \in \pi$. Then by Lemma 6 (iii), $|W|=p$.

This Lemma completes the proof of Proposition 1. However for the applications it is useful to have a slightly stronger hypothesis.

(C) Let $A_{0} \leqslant A \leqslant G$ satisfy (B) and assume that $A_{0}$ is a Hall subgroup of $A$.

LEMMA 8. Let $A_{0} \leqslant A \leqslant G$ satisfy $(C)$. Then $A$ is the centralizer of a Sylow q-subgroup of $G$ for any $q \in \pi, q \| N_{G}(A) / A \mid$. Further (i) if $\left|N_{G}(A) / A\right|$ is a $\pi^{\prime}$-group, then $A_{0}$ is a Hall subgroup of $G$, or

(ii) If $\left|N_{G}(A) / A\right|=p$ every Sylow $q$-subgroup of $A_{0}, q \in \pi, q \neq p$ is a Sylow q-subgroup of $G$.

Proof. The conclusion stated first follows from (i) and (ii). Let $R_{0}$ be a Sylow $r$-subgroup of $A_{0}, r \in \pi$. Then by Lemma $5 N_{G}\left(R_{0}\right) \leqslant N_{G}(A)$ and so if $N_{G}(A) / A_{0}$ is an $r^{\prime}$-group $R_{0}$ is a Sylow $r$-subgroup of $G$.

The situation described in Lemma 8 (ii) is the more exceptional and so it is useful to investigate it more thoroughly. (D) Let $A_{0} \leqslant A \leqslant G$ satisfy (C) and let $\left|N_{G}(A) / A\right|=p$.

Lemma 9. If $A_{0} \leqslant A \leqslant G$ satisfies (D) and $p$ is odd and the Sylow p-subgroup of $A_{0} / A_{0} \cap Z(G)$ has order greater than $p$ then $G$ has a normal $\pi$-complement.

Proof. Let $P_{0}$ be a Sylow $p$-subgroup of $A_{0}$, and let $P$ be a Sylow $p$-subgroup of $N_{G}(A)$. Then $P_{0} \triangleleft P$ and $|P| P_{0} \mid=p . \quad P_{0}$ is thus a normal Abelian maximal subgroup of $P$. If $P_{0} \neq Z J(P), P$ contains another normal Abelian maximal subgroup, say $P_{1}$. Then $P_{1} \cap P_{0}=Z(P)$. How- 
ever $\left|P_{0} / Z(P)\right| \neq p$, by assumption since $Z(P)=P \cap Z(G)$. Thus $P_{0}=Z J(P)$. Then $P$ is a Sylow $p$-subgroup of $G$ for otherwise $N_{G}\left(P_{0}\right)=N_{G}(Z J(P))$ would be greater than $N_{G}(A)$ which is false by Lemma 5 . Clearly $N_{G}(Z J(P))=N_{G}(A)$ has a normal $p$-complement and so by the ThompsonGlauberman Theorem $[5 ; 8.3 .1]$ so does $G$. Let this complement be $K$. Now $K \cap A_{0}$ is an Abelian Hall $(\pi-\{p\})$-subgroup of $G$ which is contained in the centralizer of its normalizer and so by Burnside $[6 ; 1 V, 2$, 6] the Lemma follows.

\section{Proof of Theorem 1.}

We begin by showing that Proposition 1 can be applied to the centralizer of a $\pi$-element. Let $A$ be the centralizer of a non-central $\pi$ element. So $[G: A]=n$. Further, there exists $x \in A$ such that $x$ is a $p$-element for some $p \in \pi$ and $C_{G}(x)=A$. Also, since $|\pi| \geqslant 2$, there is a non-central $q$-element $y$ say in $A$, with $q \neq p, q \in \pi$, by Lemma 1 . Now $C_{G}(x y)=C_{G}(x) \cap C_{G}(y)$ and $\left[G: C_{G}(x y)\right]=n$ and so $C_{G}(x)=C_{G}(y)=A$. Clearly if we pick any $\pi$-element of prime power order in $A$ it has order coprime to either $x$ or $y$. Thus its centralizer is either $A$ or $G$. Let $A_{0}$ be the Abelian characteristic Hall $\pi$-subgroup of $A$. We can now apply Proposition 1 to the centralizer $A$ with $A_{0}$ as the appropriate subgroup.

If $G$ is divisible by a prime $s$ which is not $G$-eccentric then the Sylow $s$-subgroup $S$ of $G$ is central. So $S$ is an Abelian direct factor of $G$. We will assume for the remainder of the proof that $|G|$ is not divisible by any primes which are not $G$-eccentric and so, in particular, that $G$ is a $\{\pi, \sigma\}$-group.

Let $x$ and $y$ be the non-central $q$-elements of $G$. Then it is clear from Lemma 8 that $C_{G}(x)$ is conjugate to $C_{G}(y)$ unless $|\pi|=2$ and $\left|N_{G}\left(C_{G}(x)\right) / C_{G}(x)\right|=p,\left|N_{G}\left(C_{G}(y)\right) / C_{G}(y)\right|=q$ for $\pi=\{p, q\}$. But from the first it could be deduced that the Sylow $q$-subgroup of $G$ is Abelian which would contradict the second statement. Let $A$ be a centralizer of some non-central $q$-element and let $A_{0}$ be the Hall $\pi$-subgroup of $A$.

Let $\omega$ be an element of a Sylow $p$-subgroup $P$ of $G p \in \pi$ such that $C_{P}(\omega) \triangleleft P$ and such that $\omega \notin Z(G)$. From Lemma 5 it follows that $P \leqslant N_{G}\left(C_{G}(\omega)\right)$. Thus $N_{G}(A)$ contains a Sylow $p$-subgroup for each $p \in \pi$. Hence $N_{G}(A)$ has $\pi^{\prime}$-index. Let $B_{0}$ be a Hall $\sigma$-subgroup of $A_{0}$, so that $A=A_{0} \times B_{0}$. Then $\exists$ a Hall $\sigma$-subgroup $H$ of $G$ such that $B_{0} \leqslant H$. 
Clearly $H \cdot N_{G}(A)=G$. Then $B_{0}^{G}=B_{0}^{N_{G}(A) H}=B_{0}^{H}$ which is a $\sigma$-group. However $H$ is nilpotent and so $B_{0} \triangleleft \triangleleft G$. Thus $B_{0} \leqslant O_{\pi^{\prime}}(G)=O_{\sigma}(G)$. So $B_{0}=O_{\pi^{\prime}}(G) \cap A$. It is clear now that $G / O_{\pi^{\prime}}(G)$ satisfies the same conditions as $G$ with $n$ replaced by $n\left|B_{0}\right| /\left|O_{\pi^{\prime}}(G)\right|$.

It will now be proved that $G$ is soluble. There are three cases.

(i) $N_{G}(A) / A$ is a $\pi^{\prime}$-group.

Then by Lemma $8, A_{0}$ is a Hall $\pi$-subgroup of $G$ and so since $G$ has a Hall $\sigma$-subgroup say $D, G=A_{0} \cdot D \quad D$ is nilpotent, $A_{0}$ is Abelian and so $G$ is soluble by Kegel-Wielandt [6; VI. 4].

(ii) $\left|N_{G}(A) / A\right|=p>2 ; p \in \pi$.

If the Sylow $p$-subgroup of $A_{0} / A_{0} \cap Z(G)$ has order $>p$, there is nothing to prove since by Lemma $9 G$ has a normal $\pi$-complement. Let $P$ be a Sylow $p$-subgroup of $N_{G}(A)$. Then $|P / P \cap Z(G)| \neq p^{2}$. Let $P_{1}$ be a Sylow $p$-subgroup of $G$ containing $P$. Let $\omega \in Z_{2}\left(P_{1}\right) \backslash Z\left(P_{1}\right)$. Then $C_{G}(\omega)$ is conjugate to $A$ and the Sylow $p$-subgroup of $N_{G}(A)$ is isomorphic to $P$. However $N\left(C_{P_{1}}(\omega)\right) \geqslant P_{1}$ and so $P_{1} \leqslant N_{G}\left(C_{G}(\omega)\right)$ by Lemma 5. Hence $P_{1}=P$. Let $X_{1}, \cdots, X_{p+1}$ be the distinct maximal subgroup of $P$ containing $Z(P)$. Now if $C_{G}\left(X_{i}\right)=C_{G}\left(X_{j}\right), i \neq j$ then $X_{i}$ and $X_{j}$ commute and so $P$ would be Abelian which is false. Hence $C_{G}\left(X_{i}\right)$ are $p+1$ distinct conjugate subgroups of $G$. Furthermore the $X_{i}$ are all conjugate since they are the Sylow $p$ subgroups of $C_{G}\left(X_{1}\right)$. Thus, as $X_{i} \triangleleft P$, $N_{G}(P) / C_{G}(P)$ is divisible by $p+1$. In particular there exists a 2-element $u$ such that $u \in N_{G}(P) \backslash C_{G}(P)$ and $u^{2} \in C_{G}(P)$. Hence $u$ normalizes some $X_{i}$. However $N_{G}\left(X_{i}\right)=N_{G}\left(C_{G}\left(X_{i}\right)\right)$ which has a normal $p$-complement and a normal Sylow 2 subgroup and hence $[u, P]=1$, contradicting the choice of $u$. This proves that this situation cannot occur.

(iii) $\left|N_{G}(A) / A\right|=2$.

Since $O_{\pi^{\prime}}(G)$ is soluble we can assume $O_{\pi^{\prime}}(G)=1$. Let $Q_{0}$ be a Sylow 2-subgroup of $A_{0}$ and let $Q$ be a Sylow 2-subgroup of $N_{G}(A)$. Then $\left|Q / Q_{0}\right|=2$. Further if $y \in Q \backslash Q_{0}, y^{2} \in X(G)$. Let $T>Z(G) \cap Q_{0}$ be such that $[T, y] \leqslant Z(G)$. Then $T \leqslant N_{G}\left(C_{G}(y)\right.$ ) by Lemma 5 . But since $\left|N_{G}\left(C_{G}(y)\right) / C_{G}(y)\right|=2$, and $C_{T}(y) \leqslant Z(G),\left|T / Z(G) \cap Q_{0}\right|=2$. Hence by [6; III. 11. 9] $Q / Z(G) \cap Q$ is a dihedral group. So the group $G / Z(G)$ has a dihedral Sylow 2-subgroup and the centralizer of an involution has an Abelian 2-complement. Thus the structure of $G / Z(G)$ is known by Gorenstein-Walter [4]. 
Assume that $K / Z(G)$ is soluble. Then there exists a normal noncentral $p$-subgroup $N$, with $p \in \pi . \quad N \leqslant N_{G}(A)$ since $\left[G: N_{G}(A)\right]$ is a $\pi^{\prime}$ number. Let $q \in \pi, p \neq q$. Then $N$ centralizes the Sylow $q$-subgroup of $A_{0}$ and so $N \leqslant A_{0}$. By Lemma $5 A \triangleleft G$ and so $G$ would be soluble.

Thus we may assume $G / Z(G)$ has no normal soluble subgroups. Then if $K / Z(G)$ is a minimal normal subgroup $K / Z(G) \cong P S L\left(2, r^{e}\right)$ of $A_{7}$ [4], where $r^{e}$ is a power of some odd prime $r$. From the hypothesis it is clear that $\sigma=\{r\}$ or $\{7\}$ respectively. Assume $K / Z(G) \cong P S L\left(2, r^{e}\right)$. Since $r^{e}>3$ we can pick $x, y$ neither being involutions such that $\mid x \| r^{e}-1$ and $\mid y \| r^{e}+1$ and so that the orders of the centralizers are not conjugate as they should be since $K \leqslant G$. Similarly for $A_{7}$ by looking at elements of order 3 and 5 .

Thus in all cases we have shown that $G$ is soluble.

Assume that $O_{\pi}(G)$ is not central. Then there exists a non-central normal $p$-subgroup $M$ of $G$, where $p \in \pi$. Then $M \leqslant N_{G}(A)$ and so $M \leqslant A$. Thus $A \triangleleft G$, from Lemma 5 . If $x$ is a non-central $\pi$-element of $G, C_{G}(x)$ is conjugate to $A$ and so $C_{G}(x)=A$. Thus $G / A$ is a $\pi^{\prime}$-group and clearly acts regularly on $A_{0} / A_{0} \cap Z(G)$. This is the situation described in (i) of Theorem 1 , note $n=[G: A]$ is a $\pi^{\prime}$-number.

Now assume that $O_{\pi}(G) \leqslant Z(G)$. However $G / O_{\pi^{\prime}}(G)$ satisfies the hypothesis for the Theorem and $O_{\pi}\left(G / O_{\pi^{\prime}}(G)\right)$ is certainly not central so we can conclude from the previous paragraph that $A O_{\pi^{\prime}}(G) \triangleleft G$, and that $A O_{\pi^{\prime}}(G)=O_{\pi^{\prime}, \pi}(G)$. Hence again $N_{G}(A) / A$ is a $\pi^{\prime}$-group and $[G: A]$ is a $\pi^{\prime}$-number. Finally if $A O_{\pi^{\prime}}(G)=L(2)$ of Theorem 1 holds by applying Lemma 3.

\section{Finite Groups of Conjugate rank 2 .}

This section is devoted to a proof of Theorem 2. We assume that $G$ is a minimal counter-example to this theorem. It follows that $G$ satisfies the following three properties:-

(i) $|G|$ is divisible by at least $3 G$-eccentric primes;

(ii) $|G|$ is divisible only by $G$-eccentric primes;

(iii) $\exists B<A<G$ with $[G: A]=n,[G: B]=m$ both $A$ and $B$ are centralizers of elements in $G$ and every element of $G$ has index $1, m$ or $n$.

Let $D \leqslant G$ and define $\pi(D)=\{p \mid p$ is a prime such that there exists a $p$-element whose centralizer is $D\} . \pi(D)$ could be empty. If $x$ is a non- 
central element of $G$ it can be classified according to the following types :

( I ) $C_{G}(x)$ is isolated; and $C_{G}(x)$ is not isolated in the remaining four cases

(II) $\quad\left[G: C_{G}(x)\right]=n,\left|\pi\left(C_{G}(x)\right)\right|>1$;

(III) $\left[G: C_{G}(x)\right]=n,\left|\pi\left(C_{G}(x)\right)\right|=1$;

(IV) $\left[G: C_{G}(x)\right]=m,\left|\pi\left(C_{G}(x)\right)\right|>1$;

(V) $\left[G: C_{G}(x)\right]=m,\left|\pi\left(C_{G}(x)\right)\right|=1$.

Since every element of $G$ has index dividing $m$, every prime dividing $|G|$ also divides $m$ by (ii).

(1) $m / n=p^{a}$ for some prime $p$ and integer $a \geqslant 1$.

From (iii) there exists an $x \in G$ such that $C_{G}(x)$ is of type (II) or (III). If $C_{G}(x)$ is of type (II) then $C_{G}(x)$ is a direct product of a nonAbelian $p$-group and an Abelian $p^{\prime}$-group and $m / n=p^{a}$ [3]. If $C_{G}(x)$ is of type (III), $m / n=p^{a}$ by the Corollary to Theorem 1 , where $\{p\}=$ $\pi\left(C_{G}(x)\right)$.

(2) There is no centralizer of type II.

Let $A$ be such a centralizer. Let $A_{0}$ be the normal Abelian Hall $p^{\prime}$-subgroup of $A$. Clearly we can apply Lemma 8 to deduce that $N_{G}(A)$ contains a Sylow $r$ subgroup of $G$, for some prime $r \neq p, r+\left[N_{G}(A): A\right]$. Since $r \mid m$ and $m=n p^{a}$ and $n=[G: A]$ this leads to a contradiction.

We can now complete the proof of the theorem. Let $X$ be a centralizer of type III. Let $\omega$ be a $p^{\prime}$-element in $X . C_{G}(\omega)$ if of type III, IV or V. Note that $C_{X}(\omega)$ is the centralizer of an element of $G$, so that if $C_{G}(\omega)$ is of type IV or $\mathrm{V}, C_{G(\omega)}=C_{X}(\omega)$. If $C_{G}(\omega)$ is of type III we would have that $m \mid n$ is of order prime to $p$ which is false. Thus the centralizer of any non-central $p^{\prime}$-element in $C_{G}(\omega)$ is precisely $C_{G}(\omega)$. From (1) and Lemma 1 we can apply Proposition 1 to $C_{G}(\omega)$ with the appropriate subgroup being the Hall $p^{\prime}$ subgroup of $C_{G}(\omega)$, the $C_{G}(\omega)$ being Abelian since it is a minimal centralizer. Finally by Lemma $8 C_{G}(\omega)$ would contain some Sylow subgroup of $G$ which is again false.

\section{REFERENCES}

[1] R. Brauer and M. Suzuki. On finite groups of even order whose 2-Sylow group is a generalized quaternion group. Proc. Nat. Acad. Sci., U.S., 45 (1959), 17571759.

[2] W. Burnside. Theory of Groups of Finite Order (Cambridge University Press). 1911. Reprinted Dover 1955. 
[ 3 ] A. R. Camina. Conjugacy Classes of finite groups and some theorems of N. Itô. J. Lond. Math. Soc., (2) 6 (1973) 421-426.

[ 4 ] D. Gorenstein \& J. H. Walter. On finite groups with dihedral Sylow 2-subgroups. Illinois J. Math., 6 (1962), 553-593.

[ 5 ] D. Gorenstein. Finite Groups. (Harper \& Row, New York). 1968.

[6] B. Huppert. Endliche Gruppen (Springer Verlag). 1967.

[ 7 ] N. Itô. On finite groups with given conjugate type I. Nagoya Math. J., 6 (1953), $17-28$.

[ 8 ] N. Itô. On finite groups with given conjugate type II. Osaka J. Math., 7 (1970), 231-251.

[ 9 ] J. Rebmann. F-Gruppen. Archiv. der Math., 22 (1971), 225-230.

[10] R. Schmidt. Zentralisatorverbande endlicher Gruppen. Rendiconti Padova, 44 (1970), 97-131.

University of East Anglia,

School of Mathematics and Physics. 